\title{
Light signaling: back to space
}

\author{
Jordi Bou-Torrent ${ }^{1}$, Irma Roig-Villanova ${ }^{1}$ and Jaime F. Martínez-García ${ }^{1,2}$ \\ ${ }^{1}$ Departament de Genètica Molecular (IBMB), Laboratori de Genètica Molecular Vegetal, Consorci CSIC-IRTA, \\ c. Jordi Girona, 18-26, 08034 Barcelona, Spain \\ ${ }^{2}$ Institució Catalana de Recerca i Estudis Avançats, Passeig Lluís Companys 23, 08010 Barcelona, Spain
}

\begin{abstract}
Recent work has increased our understanding of the molecular and cellular mechanisms of the phytochrome family of photoreceptors in controlling plant photomorphogenesis. However, the importance of long-distance communication in controlling light responses has received relatively little attention and is poorly understood. In this article, by taking a closer look at old and new experiments that extend the analysis of light signaling beyond the limits of the plant cell, we offer to look at the field in a new light. Furthermore, we discuss how intercellular and inter-organ communication might integrate with the transcriptional networks controlling lightregulated responses in plants, a novel view that might help to re-assess the parameters by which we screen for photomorphogenic mutants in the future.
\end{abstract}

\section{Phytochrome-mediated light signaling and transcriptional networks in the cell}

Light is an important positional signal that provides plants with information about their surroundings and modulates a variety of processes in their life (such as germination, seedling de-etiolation, neighbor proximity perception and flowering), collectively known as photomorphogenesis. Various photoreceptors perceive the changing light conditions and transform them into a molecular signal that eventually results in the appropriate photomorphogenic response. The phytochromes that absorb in the red $(\mathrm{R})$ and far-red (FR) region of light spectrum are among the bestcharacterized photoreceptors. Much of our understanding of phytochrome-mediated photomorphogenesis comes from analyses of seedling de-etiolation and shade avoidance syndrome (SAS) responses in Arabidopsis thaliana. However, molecular models describing the action of phytochromes and interacting partners are often restricted to single cells. The obvious fact that these regulatory networks are in the multicellular context of the plant, where not all the cells are expressing the same genes at the same time, has received relatively little attention. In addition, the sites of light perception and light action do not always overlap in the plant, highlighting the importance of longdistance communication to understand fully how light controls plant growth and development. The central importance of the spatial control of photomorphogenesis has been recently recognized in reviews on light-regulated development in plants [1,2]. Recent findings, which demonstrate that light perceived in leaves can regulate development of distant organs via changes in hormone signaling

Corresponding author: Martínez-García, J.F. (jmggmg@ibmb.csic.es).
[3] or transport of protein molecules [4], have brought to our attention data generated decades ago about how different organs perceive, integrate and respond to light signals [5-13]. Here, we aim to renew interest in understanding the spatial dimension of light-regulated plant development by reformulating old questions (Box 1). By focusing on what is known about phytochrome regulation of seedling deetiolation and SAS responses as paradigms of light-signaling, we will outline answers to some of these questions using current concepts. Additionally, our considerations might help to re-assess the criteria used to screen for photomorphogenic mutants in the future.

\section{Light signaling: from cell to seedling}

In a strict sense, signaling refers to events from signal perception to the first changes in gene expression elicited by the signal (i.e. pre-transcriptional events or immediate interactions following signal recognition) [14]. In the plant field, however, this term is frequently used more loosely [15], encompassing events from light perception to the appearance of measurable growth or developmental changes. In the context of a whole plant, light signaling involves at least two steps (Figure 1): (i) intracellular signaling, by definition a cell-autonomous step triggered by the phytochrome molecules within the cell upon perception of the light signal, and (ii) intercellular signaling, when the measurable changes in growth and development occur in a place that is distinct from the cells or tissue that received the light stimulus. These steps will be analyzed in detail in the following sections.

Intracellular light signaling: how light might inhibit cell growth in the hypocotyl, but promote it in the cotyledons

In the cellular context, light signaling includes events from light perception to the appearance of cellular changes (gene expression, cell elongation or expansion, chloroplast movement, etc.). Although light is perceived by at least three types of photoreceptors, only phytochromes will be covered here. In the dicot plant Arabidopsis thaliana, phytochromes are encoded by a small gene family of five members (PHYA-PHYE). By contrast, in monocots, this family contains only three members (PHYA-PHYC) [16,17]. The different phytochromes have unique and overlapping biological functions $[1,16,18]$. They exist in two photoreversible conformations: the R-light-absorbing Pr form, biologically inactive, and the FR-light-absorbing Pfr form, biologically active. In dark-grown (etiolated) seedlings, phytochromes are cytosolic inactive $(\mathrm{Pr})$ proteins 


\section{Box 1. Old questions to be answered with new eyes}

- What mechanisms allow light to inhibit cell growth in the hypocotyl but promote it in the cotyledons?

- When does the ubiquitous phytochrome signal become cell or organ specific?

- How might light responses communicate and coordinate between organs, such as cotyledons (or leaves) and hypocotyls (or stems)?

(Figure 1a). Following light exposure, phytochromes rapidly convert to Pfr and migrate to the nucleus, where they orchestrate a complex transcriptional cascade through their interaction with different phytochrome interacting factors (PIFs) [18,19]. This transcriptional network is instrumental in implementing the appropriate developmental program that leads to cotyledon expansion and greening, root growth and decreased hypocotyl elongation, eventually resulting in seedling de-etiolation (Boxes 2,3). Once the plant is de-etiolated, the photo-equilibrium between inactive and active nuclear phytochromes is established. Reductions in the R/FR ratio associated with plant proximity or canopy shade displace the photo-equilibrium towards the inactive Pr form, globally affecting the phytochrome-controlled transcriptional cascade. As a consequence, growth is reoriented to implement SAS responses such as increased stem and petiole growth, reduced branching and flowering induction that instigate competition with other plants for light [2,18]. A question arising from these different outcomes of phytochrome action is how light might inhibit growth in one organ but promote it in another.

The different responses to light in different tissues are likely to be the result of a combination of factors. Physiological differences among cells or organs (e.g. pigmentation due to photosynthetic activity and metabolic status) might influence light sensitivity. Although spatial expression analyses have led to the conclusions that phytochromes are found in all tissues analyzed (even in roots) and that most plant cells contain phytochromes and, therefore, are $\mathrm{R}$ - and FR-sensitive [20], the distribution of phytochromes and interacting partners in a given tissue or organ might not be homogeneous. As a consequence, the spatial expression patterns of early targets of phytochrome action might be tissue-specific. Indeed, data extracted from a recent analysis of transcript profiles in roots, hypocotyls and cotyledons of Arabidopsis seedlings [21] reveal that the

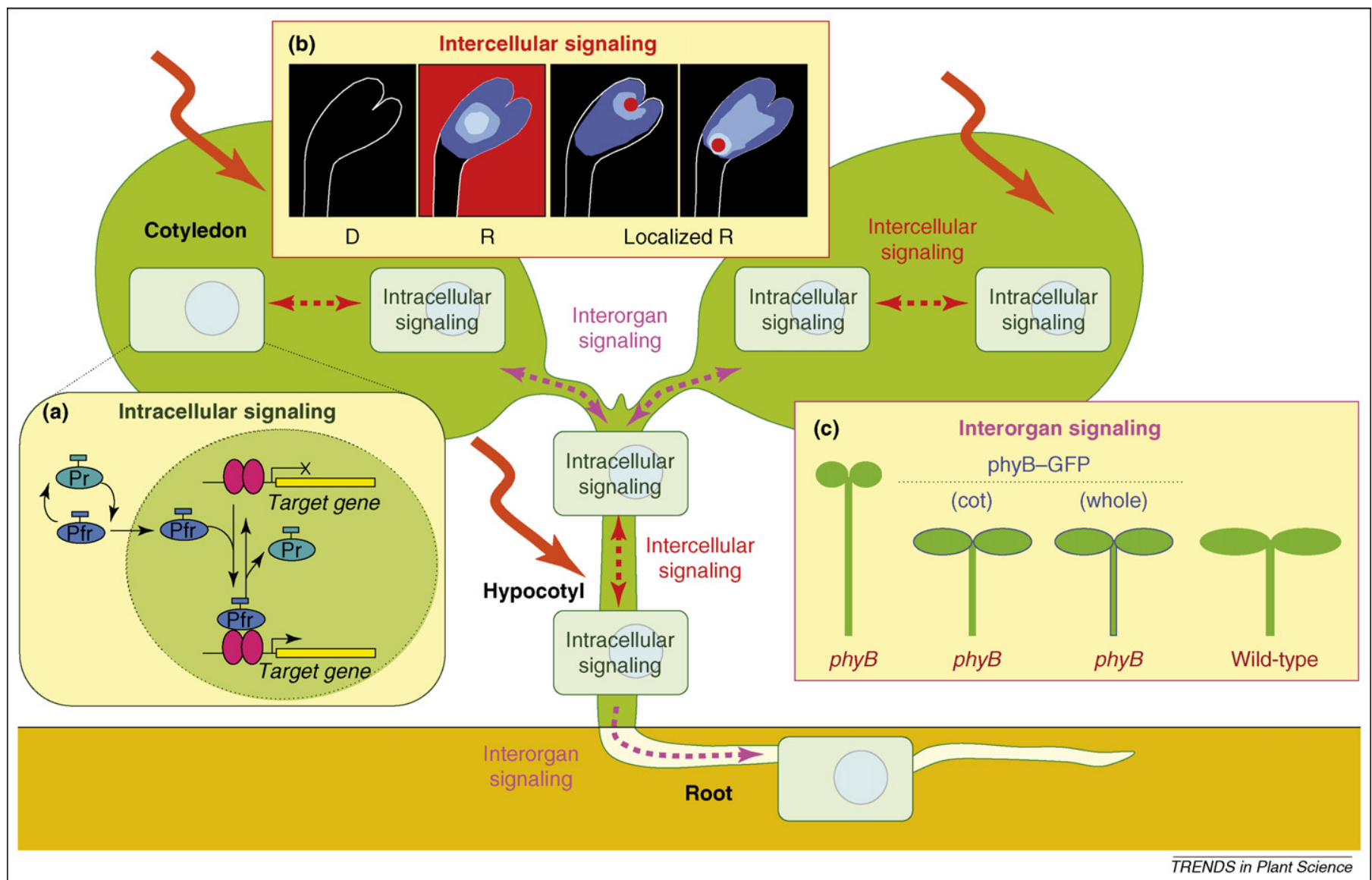

Figure 1. Model representing the web of interactions following light (red arrow) perception in the context of the whole seedling. (a) Within a cell, light perception by the phytochromes establishes their photo-equilibrium between inactive (Pr) and active (Pfr) forms and induces nuclear translocation; there, phytochromes might bind to PIFs (red circles) in a photo-reversible manner, affecting expression of target genes. This process initiates intracellular signaling in the cells of exposed organs by modulating a transcriptional network. The figure is based on Ref. [19]. (b) The existence of intercellular signaling was shown with experiments like the one depicted in the cartoon [13]. Upon illumination of dark-grown transgenic seedlings expressing a luciferase reporter under the control of a light-induced gene with red ( $R$ ) light or a localized $\mathrm{R}$ irradiation (red dot), the presence of luciferase activity was found in the areas depicted with blue coloring (light blue = highest; dark blue = lowest; black = no response) within domains of the cotyledons. (c) Inter-organ signaling was neatly illustrated by experiments in which the fusion protein phyB-GFP complemented the long hypocotyl phenotype of phyB-deficient seedlings ( $p h y B$ ) when overexpressed only in the cotyledons (cot) or in the whole seedling [24]. These experiments support the existence of diffusible signal(s) of unknown nature (dotted arrows) produced by the light-stimulated cells and transmitted to (b) adjacent cells (intercellular signaling) or (c) distant organs (interorgan signaling). 
Box 2. Seedling de-etiolation: the sum of individual and coordinated changes

Seedling de-etiolation refers to the changes in development triggered by the first exposure of seedlings to light after germination. Dark-grown seedlings display rapidly elongating hypocotyls, closed and unexpanded cotyledons of pale-yellow color (due to the low abundance of carotenoids and absence of chlorophylls), apical hooks enclosing undeveloped leaf primordia and short roots. After transfer to light, hypocotyl growth is arrested, the apical hook is straightened, cotyledons expand and unfold, the root elongates and the seedling greens. The morphological changes are concomitant with at least two connected traits: gene expression and metabolism. Changes in gene expression, which can be early or late, transitory or permanent, and positive or negative, are part of a complex transcriptional network, hypothesized to be instrumental for the implementation of the metabolic and morphologic changes. In the seedling context, the transcriptional network might diverge spatially, resulting in metabolic and morphological organ-specific changes.

Are these morphological, molecular and metabolic changes connected? Physiological and genetic analyses give some clues about their relationship. Wild-type seedlings grown under continuous FR (FRc) and photomorphogenic mutants of the COP-DETFUS group grown in darkness display obvious de-etiolated phenotypes and show high expression levels of light-regulated genes in darkness, although none of them accumulate chlorophyll $[1,18]$. Some hormone mutants also show a de-etiolated phenotype in darkness (including cotyledon development) and accumulate high transcript levels of light-regulated genes. This is the case of the auxin signal transduction axr1 mutants, the brassinosteroid biosynthetic mutants $c p d$, det2 and $d w f 4$ or the gibberellin mutants ga1, gai and na [44-48]. It has been proposed that the expression of lightregulated genes is a consequence of leaf (cotyledon) development rather than light exposure [49]. In addition, transcript levels of genes such as $A T H B 2, P A R 1$ and PIL1, which are rapidly downregulated by light, are not altered in dark-grown cop1 mutant seedlings unless they are illuminated [22]. Together, these data show that some of the morphologic, metabolic and molecular changes triggered by light can be uncoupled, suggesting that, in some cases, they might be regulated independently.

expression patterns of $P H Y A, P H Y B$, several $P I F \mathrm{~s}$ and a few early targets of phytochrome action during both deetiolation and SAS (e.g. ATHB2, ATHB4, HAT2, PAR1, $P I L 1, R I P)$ [22] differ in response to light in the three organs analyzed in both etiolated and light-grown seedlings (Figure 2). It is therefore tempting to speculate that phytochrome perception and transduction systems vary among the different organs. As a result, upon light perception, the transcriptional network might immediately diverge in a cell- and organ-specific manner, eventually resulting in different, even opposing, organ responses to the same light stimulus.

\section{Intercellular light signaling within the same organ: when non-irradiated or distant cells respond to light perception}

The existence of diffusible signals generated by the light stimulus in irradiated cells and transmitted to distant responsive cells (irradiated or not) within the same organ was deduced decades ago from physiological experiments. For instance, isolated cowpea (Vigna sinensis) epicotyl (first internode) explants elongate in response to end-ofday FR (EOD-FR) treatments, which can be considered a SAS response [7]. Within the epicotyl, the EOD-FR-responsive region spans a section $5-20 \mathrm{~mm}$ below the apex [10].

\section{Box 3. The classical concept of the 'photomorphogenic mutant'}

Light induces concomitant and reciprocal responses in hypocotyls (inhibition of elongation) and cotyledons (stimulation of expansion). These opposite responses, used as a diagnostic marker of lightregulated development, have helped to originate the concept of the 'photomorphogenic mutant'.

In the case of the identification of phytochrome-signaling components, most of the direct genetic screens for mutants initially relied on the ease of scoring the response of hypocotyl elongation to continuous FR (FRc) or R (Rc). The simultaneous occurrence of opposed growth of the cotyledons has since been used to classify the isolated mutants as truly photomorphogenic. This classification was intended to distinguish them from other mutants that are globally affected in growth responses per se, such as overall dwarf or gigantic seedlings. Whereas photomorphogenic mutants, by definition, are supposed to affect specifically early light-signaling events, the latter mutants are expected to affect downstream steps, such as those involving plant hormones [15]. Although this criterion has generally worked very well, its strict application might lead to paradoxical conclusions.

One such paradox is the red elongated1 (red1) mutant, which was originally identified as a phyB signaling mutant (i.e. photomorphogenic) because of its exclusive hyposensitivity to Rc (longer hypocotyls and reduced cotyledon expansion in Rc, but not in FRc) compared with wild-type seedlings [50]. Cloning of RED1 revealed that it actually encodes a modifying enzyme involved in auxin deactivation, which suggests that the red1 mutation results in auxin overproduction rather than the alteration of an early lightsignaling event [51]. Therefore, in spite of its obvious photomorphogenic phenotype, red1 seedlings are paradoxically authentic hormone mutants.

On the other hand, components clearly involved in photomorphogenic development can generate a 'non-photomorphogenic' phenotype when mis-expressed. For instance, light-grown cop1 and det1 seedlings $[1,18]$ are dwarf with shorter hypocotyls and lessexpanded cotyledons than the wild type [52]. Overexpression of PHYB also results in dwarf plants with less-expanded leaves [53]. These parallel, unidirectional defects in both hypocotyl (stem) and cotyledon (leaf) growth would suggest that they are the result of global defects in cell elongation and expansion, despite the wellestablished role of phyB, COP1 and DET1 in photomorphogenesis.

Excision of the upper $5 \mathrm{~mm}$ of the epicotyl strongly decreases the growth response of the adjacent 5 to $20-\mathrm{mm}$ region to the EOD-FR treatments. This surgical treatment, however, does not reduce (but slightly increases) the elongation response of the remaining epicotyl to exogenously applied gibberellin $\mathrm{A}_{1}$, a plant hormone known to stimulate epicotyl elongation [10]. This result indicated that the missing upper 5-mm region, although less responsive to the EOD-FR treatment, contributes to the light-induced elongation of the 5 to $20-\mathrm{mm}$ region without decreasing the ability of the organ to respond to other stimuli. This evidence supports the existence of a signal integration network with a final output that is the sum of the individual and coordinated activities of the cells that form it.

The existence of intercellular light signaling was elegantly demonstrated by localized irradiation of small areas within the cotyledon of tobacco and mustard etiolated seedlings $[12,13]$. In etiolated tobacco seedlings expressing a LUCIFERASE (LUC) reporter gene under the control of the light-responsive $C A B 2$ gene promoter $(C A B 2:: L U C)$, luciferase activity was observed throughout the cotyledon after exposure of the whole organ to light [13]. When only a few cells were irradiated with a microbeam, luciferase 


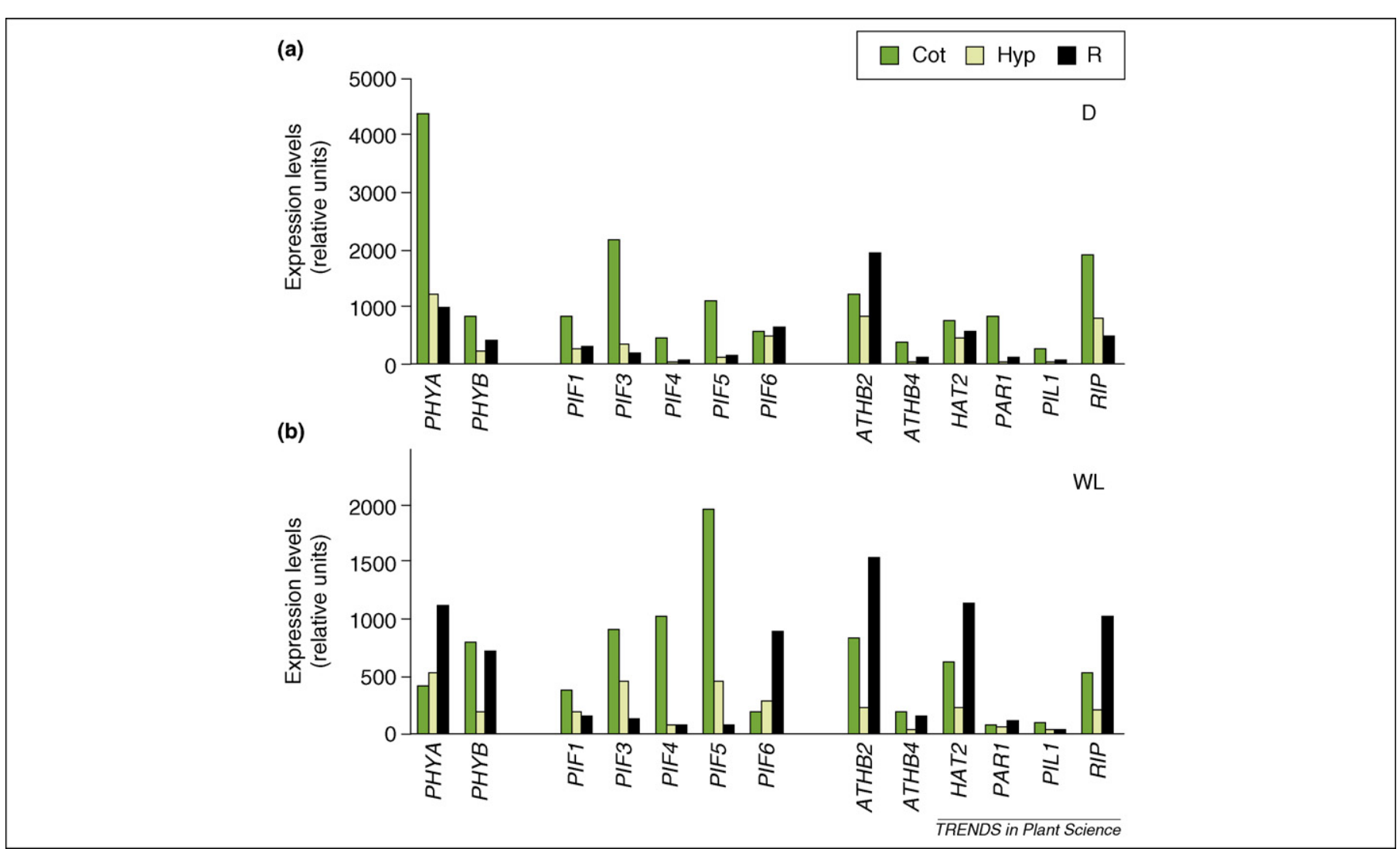

Figure 2. Phytochromes, PIFs and early targets of phytochrome action are differentially expressed in seedling organs. Expression levels of $P H Y A, P H Y B$, several $P I F \mathrm{~S}(P I F 1$, PIF3-PIF6) and early targets of phytochrome action during both de-etiolation and SAS (ATHB2, ATHB4, HAT2, PAR1, PIL1, RIP) [22] in different organs from seedlings grown under (a) dark or (b) white-light conditions are based on data from recent microarray experiments [21]. Abbreviations: Cot, cotyledon; Hyp, hypocotyl; R, root.

activity was detected not only in the irradiated cells, but also in areas of the cotyledon that had not received the light stimulus (Figure 1b). Non-irradiated cells of mustard cotyledons also showed a clear response to microbeam stimulation of distant cells [12], again confirming the existence of intercellular signaling. The biological significance for this intercellular and intra-organ light signaling might be to ensure a coordinated, optimal and global organ response by averaging (buffering) the local differences in the amount or spectral distribution of the light stimulus.

\section{Inter-organ light signaling: how light responses might} communicate and coordinate between organs

There are many data supporting a communication between different organs in the regulation of different plant photoresponses, such as seedling de-etiolation, SAS, branching and flowering [3-6,8,11,23,24]. A few illustrative examples will be described here.

\section{De-etiolation}

In etiolated cucumber seedlings, hypocotyl growth is strongly inhibited by continuous $\mathrm{R}$ or FR light [5]. Darkening the cotyledons with aluminum foil reduces the inhibitory effect of $\mathrm{R}$ light, but not of FR light, in hypocotyl growth [5], which suggests that the main FR-light receptor phyA perceives and signals in the hypocotyl itself whereas cotyledon-localized phyB, the main R-light receptor, can send a signal to the elongating hypocotyl. In Arabidopsis, expression of the fusion protein phyB-GFP only in cotyledons can complement the long hypocotyl phenotype of phyB-deficient seedlings $($ phyB) grown under white light [24] (Figure 1c). These results confirm that $\mathrm{R}$-light perception in the cotyledons can be transduced as diffusible signals to inhibit growth in a distant organ, the hypocotyl.

\section{SAS responses}

In mustard, epicotyl elongation is induced after irradiating the whole seedling with white light supplemented with FR [6,8] or EOD-FR treatments [11] to simulate shade. However, localized FR irradiation of only the primary leaves also results in growth of the epicotyl $[6,8,11]$. Similarly, when transgenic Arabidopsis lines that show $\beta$-glucuronidase (GUS) activity specifically in the hypocotyl after EODFR treatments were used for localized irradiation of the cotyledons, GUS expression was clearly detected in the hypocotyls [23]. Furthermore, an auxin transport inhibitor reduced EOD-FR-dependent GUS activity in the hypocotyls of some of the lines analyzed, suggesting the involvement of auxin in the transmission of a shade-related signal originated in the irradiated cotyledons [23]. Taken together, these results highlight the importance of cotyledons and leaves for shade-controlled elongation of separated organs (hypocotyls and epicotyls) in some species. This effect, however, is not conserved in the plant kingdom because there are examples of plant species in which localized treatment of leaves with simulated shade does not stimulate stem elongation [7]. 


\section{Branching}

There is recent evidence in support of shoot-to-root crosstalk in Arabidopsis, where it was shown that shoot-localized phytochromes are able to act over long distances to regulate the emergence of lateral roots [3]. This phytochrome effect is proposed to happen, at least in part, through modulation of auxin transport and sensitivity [3]. The development of shoot secondary meristems leading to branches is also influenced by light, probably perceived in the leaves and controlled by genes expressed in distant cells of axillary buds [17,25-27]. Auxins, that also appear to be involved in long-distance signaling controlling shoot branching, might serve as transducers of light signals to regulate axillary branch development, as part of the SAS responses in grasses [17]. Additionally, signals derived from roots have also been shown to regulate shoot branching. Isolation of Arabidopsis MORE AXILLARY BRANCHES $(M A X)$ mutants and related mutants in pea and petunia have shown that hormone-like carotenoidderived signals produced in the root can be transported and influence branching in shoot tissues $[17,28]$. Therefore, hormone signals appear to be key components of longrange communication between organs in photomorphogenesis.

\section{Flowering}

In several species, perception of day-length (photoperiod) and plant proximity (canopy shade) in the leaves is an important signal controlling flower induction in the shoot apex. These two physically separated processes are connected by the elusive florigen, a signal generated in the leaves under inductive photoperiods and translocated from leaves to the shoot apex [4,17]. Recently, it has been concluded that the protein FLOWERING LOCUS T (FT) itself constitutes a major component of the florigen [29-32] Consistently, phyB-GFP production only in leaf mesophyll cells rescues the early flowering phenotype of the Arabidopsis phyB mutants, probably by locally suppressing $F T$ expression [24]. It is interesting to note that this is a mechanism of long-distance signaling distinct from others of hormonal nature.

In summary, the global organ response to a light stimulus requires the integration of the individual cellular photo-responses with intercellular and inter-organ signals that connect and coordinate them. Although the nature of these signals is unknown in most cases, other hormones in addition to auxins are known to participate in locally controlling light responses, such as gibberellins [33] and cytokinins [34], making hormones good candidates to act as integrators of light responses [35,36]. Indeed, mutants initially classified as photomorphogenic based on their phenotype were later classified as hormone biosynthetic mutants after identifying the mutated gene (Box 3). The reported inter-organ mobility of macromolecules, such as small RNAs, mRNAs and proteins [4,37], might make this list much more complex.

\section{Intercellular and inter-organ light signaling: future perspectives}

The phenotypic characterization of mutants defective in specific light responses has been used to define components of photomorphogenic networks. For instance, loss-of-function mutations in components intimately associated with the photoactive phytochromes were expected to have a classical photomorphogenic phenotype, showing hypo- or hypersensitivity to the light stimulus and to affect any of several steps from chromophore biosynthesis, phytochrome photoactivation, nuclear translocation, affinity for downstream transducers and/or access to gene promoters. This is actually the case for mutants such as hy1, hy2, fhy1, ndpk2, ppap5 or pif3 [1,38-40]. But, as described here, light signaling also involves cell-to-cell and longdistance communication. Although the identification of mutants impaired in these processes would be key in clarifying the molecular mechanisms involved, it is not clear if such mutants would be 'photomorphogenic' according to classical standards (Box 3). To illustrate this point, we will focus on a recent work on the functional characterization of early targets of phytochromes during seedling de-etiolation [41].

The role of a set of 32 genes previously identified as being rapidly regulated by phytochromes was analyzed by reverse genetics [41]. The authors applied the classical photomorphogenic concept (Box 3) to discriminate a set of only seven mutants specifically controlling seedling de-etiolation (class I). This mutant-class I, which displays opposed growth responses of hypocotyl and cotyledons to light, might represent components closely affecting phytochrome action. Examples of these components are PHYTOCHROME KINASE SUBSTRATE1 (PKS1), previously shown to interact with phytochromes [42], and PIF3-LIKE1 (PIL1), a basic-helix-loop-helix factor that might affect phytochrome signaling by interacting with other PIFs, as has been shown for members of this subgroup [1].

Two additional phenotypic classes were also described: those having parallel, unidirectional defects in both hypocotyl and cotyledon photo-responsiveness (class II, seven genes) and those displaying significant deviations in lightregulated growth of only the hypocotyls (class III, six genes). Class II was considered to be globally defective in cell elongation and expansion processes (e.g. hormonal mutants) and, therefore, disrupted class II genes were not considered to be specifically involved in the light-signaling pathways (i.e. not necessarily photomorphogenic). Class III was considered to represent organ-specific components involved in either normal phytochrome signaling or more general cell-growth processes [41]. However, all three classes can be considered light-signaling mutants because expression of the corresponding genes is regulated by phytochromes soon after signal perception [15]. A corollary of this reinterpretation is that light-signaling mutants do not necessarily have to show concomitant and reciprocal light-induced defects in both hypocotyl and cotyledon growth (Box 3). For instance, some of the genes from classes II and class III [41] might correspond to ubiquitous and/or tissue-specific components involved in coordinating growth among different organs during light-triggered seedling de-etiolation. In summary, such mutants might help to redefine what is to be expected from altering the spatial dimension of light signaling, assigning a function to novel or known photomorphogenic components in the steps 
involved in transforming the intracellular phytochrome signal into a global seedling photo-response.

Future approaches to understand better the spatial aspects of photomorphogenesis will probably involve the generation of transgenic plants with an organ-specific overexpression of candidate genes. The studies describing the Arabidopsis FT protein and its rice ortholog Hd3a as the long-distance signal that induces flowering [29-32] provide elegant strategies to address inter-organ communication, which could eventually be useful in the study of light signaling. The information generated might help to improve crop performance through the modification of light responses in specific tissues or organs, reducing the detrimental and pleiotropic effects observed when manipulating phytochrome in the whole plant $[17,43]$.

\section{Acknowledgements}

We are very grateful to Jordi Casanova, Pep Casacuberta, Paul Devlin, José Luis García-Martínez and Manuel Rodríguez-Concepción for critical reading and comments on the manuscript. JB-T was the recipient of a postdoctoral contract from the Generalitat de Catalunya. IR-V was the recipient of a predoctoral fellowship from the FPI program. Research in our laboratory is supported by grants from the Spanish Ministry of Science and Education - FEDER to JFM-G (BIO2005-00154). We are part of the 'Grup de Recerca Emergent' 2005SGR 00284 and 'Xarxa de Referència en Biotecnologia'.

\section{References}

1 Chen, M. et al. (2004) Light signal transduction in higher plants. Annu. Rev. Genet. 38, 87-117

2 Jiao, Y. et al. (2007) Light-regulated transcriptional networks in higher plants. Nat. Rev. Genet. 8, 217-230

3 Salisbury, F.J. et al. (2007) Phytochrome coordinates Arabidopsis shoot and root development. Plant J. 50, 429-438

4 Kobayashi, Y. and Weigel, D. (2007) Move on up, it's time for changemobile signals controlling photoperiod-dependent flowering. Genes Dev. 21, 2371-2384

5 Black, M. and Shuttleworth, J.E. (1974) The role of the cotyledons in the photocontrol of hypocotyl extension in Cucumis sativus L. Planta $117,57-66$

6 Morgan, D.C. et al. (1980) Rapid photomodulation of stem extension in light-grown Sinapis alba L. Planta 150, 95-101

7 García-Martínez, J.L. et al. (1987) Phytochrome regulation of the response to exogenous gibberellins by epicotyls of Vigna sinensis. Plant Physiol. 85, 212-216

8 Casal, J.J. and Smith, H. (1988) Persistent effects of changes in phytochrome status on internode growth in light-grown mustard: Occurrence, kinetics and locus of perception. Planta 175, 214-220

9 Ballaré, C.L. et al. (1990) Far-red radiation reflected from adjacent leaves: an early signal for competition in plant canopies. Science 247 , 329-332

10 Martínez-García, J.F. and García-Martínez, J.L. (1992) Interaction of gibberellins and phytochrome in the control of cowpea epicotyl elongation. Physiol. Plant. 86, 236-244

11 Yanovsky, M.J. et al. (1995) Are phytochrome-mediated effects on leaf growth, carbon partitioning and extractable sucrose-phosphate synthase activity the mere consequence of stem-growth responses in light-grown mustard? J. Exp. Bot. 46, 753-757

12 Nick, P. et al. (1993) Cell communication, stochastic cell responses, and anthocyanin pattern in mustard cotyledons. Plant Cell 5, 541-552

13 Bischoff, F. et al. (1997) Phytochrome-induced intercellular signaling activates cab:luciferase gene expression. Plant J. 12, 839-849

14 McCarty, D.R. and Chory, J. (2000) Conservation and innovation in plant signaling pathways. Cell 103, 201-209

15 Quail, P.H. (2006) Signal transduction in photomorphogenesis. General introduction. In Photomorphogenesis in Plant and Bacteria (3rd edn) (Schäfer, E. and Nagy, F., eds), pp. 329-334, Springer

16 Takano, M. et al. (2005) Distinct and cooperative functions of phytochrome A, B, and $\mathrm{C}$ in the control of deetiolation and flowering in rice. Plant Cell 17, 3311-3325
17 Kebrom, T.H. and Brutnell, T.P. (2007) The molecular analysis of the shade avoidance syndrome in the grasses has begun. J. Exp. Bot. 58, 3079-3089

18 Quail, P.H. (2002) Phytochrome photosensory signalling networks. Nat. Rev. Mol. Cell Biol. 3, 85-93

19 Martínez-García, J.F. et al. (2000) Direct targeting of light signals to a promoter element-bound transcription factor. Science 288, 859863

20 Sharrock, R.A. and Mathews, S. (2006) Phytochrome genes in higher plants: structure, expression, and evolution. In Photomorphogenesis in Plant and Bacteria (3rd edn) (Schäfer, E. and Nagy, F., eds), pp. 99129 , Springer

$21 \mathrm{Ma}$, L. et al. (2005) Organ-specific expression of Arabidopsis genome during development. Plant Physiol. 138, 80-91

22 Roig-Villanova, I. et al. (2006) Identification of primary target genes of phytochrome signaling. Early transcriptional control during shade avoidance responses in Arabidopsis. Plant Physiol. 141, 85-96

23 Tanaka, S. et al. (2002) Phytochrome in cotyledons regulates the expression of genes in the hypocotyl through auxin-dependent and independent pathways. Plant Cell Physiol. 43, 1171-1181

24 Endo, M. et al. (2005) Phytochrome B in the mesophyll delays flowering by suppressing FLOWERING LOCUS T expression in Arabidopsis vascular bundles. Plant Cell 17, 1941-1952

25 Reed, J.W. et al. (1993) Mutations in the gene for the red/far-red light receptor phytochrome $\mathrm{B}$ alter cell elongation and physiological responses throughout Arabidopsis development. Plant Cell 5, 147157

26 Kebrom, T.H. et al. (2006) Phytochrome B represses teosinte branched 1 expression and induces sorghum axillary bud outgrowth in response to light signals. Plant Physiol. 140, 1109-1117

27 Takeda, T. et al. (2003) The OsTB1 gene negatively regulates lateral branching in rice. Plant J. 33, 513-520

28 Mouchel, C.F. and Leyser, O. (2007) Novel phytohormones involved in long-range signaling. Curr. Opin. Plant Biol. 10, 473-476

29 Corbesier, L. et al. (2007) FT protein movement contributes to longdistance signaling in floral induction of Arabidopsis. Science 316, 1030 1033

30 Jaeger, K.E. and Wigge, P.A. (2007) FT protein acts as a long-range signal in Arabidopsis. Curr. Biol. 17, 1050-1054

31 Mathieu, J. et al. (2007) Export of FT protein from phloem companion cells is sufficient for floral induction in Arabidopsis. Curr. Biol. 17, $1055-1060$

32 Tamaki, S. et al. (2007) Hd3a protein is a mobile flowering signal in rice. Science 316, 1033-1036

33 Martínez-García, J.F. et al. (2000) The end-of-day far-red irradiation increases gibberellin A1 content in cowpea (Vigna sinensis) epicotyls by reducing its inactivation. Physiol. Plant. 108, 426-434

34 Carabelli, M. et al. (2007) Canopy shade causes a rapid and transient arrest in leaf development through auxin-induced cytokinin oxidase activity. Genes Dev. 21, 1863-1868

35 Morelli, G. and Ruberti, I. (2002) Light and shade in the photocontrol of Arabidopsis growth. Trends Plant Sci. 7, 399-404

36 Halliday, K.J. and Fankhauser, C. (2003) Phytochrome-hormonal signaling networks. New Phytol. 157, 449-463

37 Lough, T.J. and Lucas, W.J. (2006) Integrative plant biology: role of phloem long-distance macromolecular trafficking. Annu. Rev. Plant Biol. 57, 203-232

$38 \mathrm{Kim}$, J. et al. (2003) Functional characterization of phytochrome interacting factor 3 in phytochrome-mediated light signal transduction. Plant Cell 15, 2399-2407

39 Hiltbrunner, A. et al. (2005) Nuclear accumulation of the phytochrome A photoreceptor requires FHY1. Curr. Biol. 15, 2125-2130

40 Ryu, J.S. et al. (2005) Phytochrome-specific type 5 phosphatase controls light signal flux by enhancing phytochrome stability and affinity for a signal transducer. Cell 120, 395-406

41 Khanna, R. et al. (2006) Functional profiling reveals that only a small number of phytochrome-regulated early-response genes in Arabidopsis are necessary for optimal deetiolation. Plant Cell 18, 2157-2171

42 Fankhauser, C. et al. (1999) PKS1, a substrate phosphorylated by phytochrome that modulates light signaling in Arabidopsis. Science $284,1539-1541$

43 Sawers, R.J.H. et al. (2005) Cereal phytochromes: targets of selection, targets for manipulation? Trends Plant Sci 10, 138-143 
44 Schwechheimer, C. et al. (2002) Multiple ubiquitin ligase-mediated processes require COP9 signalosome and AXR1 function. Plant Cell 14, $2553-2563$

$45 \mathrm{Li}$, J. et al. (1996) A role for brassinosteroids in light-dependent development of Arabidopsis. Science 272, 398-401

46 Szekeres, M. et al. (1996) Brassinosteroids rescue the deficiency of CYP90, a cytochrome P450, controlling cell elongation and deetiolation in Arabidopsis. Cell 85, 171-182

47 Azpiroz, R. et al. (1998) An Arabidopsis brassinosteroid-dependent mutant is blocked in cell elongation. Plant Cell 10, 219-230

48 Alabadí, D. et al. (2004) Gibberellins repress photomorphogenesis in darkness. Plant Physiol. 134, 1050-1057

49 Chory, J. et al. (1989) Arabidopsis thaliana mutant that develops a light-grown plant in the absence of light. Cell 58, 991-999
50 Wagner, D. et al. (1997) RED1 is necessary for phytochrome B-mediated red light-specific signal transduction in Arabidopsis. Plant Cell 9, 731-743

51 Hoecker, U. et al. (2004) The photomorphogenesis-related mutant red1 is defective in CYP83B1, a red light-induced gene encoding a cytochrome P450 required for normal auxin homeostasis. Planta $219,195-200$

52 McNellis, T.W. et al. (1994) Genetic and molecular analysis of an allelic series of cop1 mutants suggests functional roles for the multiple protein domains. Plant Cell 6, 487-500

53 Thiele, A. et al. (1999) Heterologous expression of Arabidopsis phytochrome $\mathrm{B}$ in transgenic potato influences photosynthetic performance and tuber development. Plant Physiol. 120, 7382

\section{Have you contributed to an Elsevier publication? Did you know that you are entitled to a $30 \%$ discount on books?}

A $30 \%$ discount is available to all Elsevier book and journal contributors when ordering books or stand-alone CD-ROMs directly from us.

To take advantage of your discount:

\section{Choose your book(s) from www.elsevier.com or www.books.elsevier.com}

\section{Place your order}

Americas:

Phone: +18007824927 for US customers

Phone: +18004603110 for Canada, South and Central America customers

Fax: +13144534898

author.contributor@elsevier.com

All other countries:

Phone: $+44(0) 1865474010$

Fax: +44 (0)1865 474011

directorders@elsevier.com

You'll need to provide the name of the Elsevier book or journal to which you have contributed. Shipping is free on prepaid orders within the US.

If you are faxing your order, please enclose a copy of this page.

\section{Make your payment}

This discount is only available on prepaid orders. Please note that this offer does not apply to multi-volume reference works or Elsevier Health Sciences products.

For more information, visit www.books.elsevier.com 